\title{
Eritrodermia con bacteriemia por Streptococcus dysgalactiae subespecie equisimilis en un paciente pediátrico: reporte de un caso y revisión de la literatura
}

\author{
Miguel A. Pantoja, Luis Delpiano y Gia Haquin
}

\section{Streptococcus dysgalactiae subspecies equisimilis bacteremic erythroderma in a pediatric patient: a case report and review of literature}

Infections caused by pyogenic streptococci are commons in pediatric ages. However, in the last decades there has been an increase in the isolation of no A or B Streptococci. We report a case of a 6 years old girl, who presents fever for 5 days and erythroderma. Streptococcus dysgalactiae subspecies equisimilis was isolated from blood cultures. She receives antibiotics for 14 days with $\beta$-lactams with a good clinic evolution with normalization of the inflammatory parameters. This agent $\beta$-hemolítico presents antigens of Lancefield groups A, C and G, and a great similitude regarding virulence factors, with Streptococcus pyogenes. Frequently in old patients, few reported cases in pediatric population. First line treatment remains $\beta$-lactam antibiotics for which there are no reports of increasing resistance.

Key words: Streptococcus dysgalactiae, streptococcal infections, soft tissue infections, bacteremia.

Palabras clave: Streptococcus dysgalactiae, infección estreptocóccica, infección de tejidos blandos, bacteriemia.

\section{Introducción}

S treptococcus $\beta$-hemolíticos son cocáceas grampositivas con actividad hemolítica intensa en agar sangre. La antigua clasificación en grupos de Lancefield ya está en desuso pues reunía bacterias con características muy distintas, sólo por poseer un antígeno de superficie en común. Dentro de este grupo, están los llamados estreptococos piógenos, que son una causa frecuente de infecciones, tanto en pacientes pediátricos como adultos ${ }^{1}$.

Los principales estreptococos $\beta$-hemolíticos patógenos son Streptococcus pyogenes (Grupo A de Lancefield) y Streptococcus agalactiae (Grupo B). Sin embargo, en las últimas décadas, y con el perfeccionamiento de los métodos diagnósticos, se ha observado un aumento en la frecuencia de infecciones por Streptococcus $\beta$-hemolíticos no A ni B, dentro de los cuales destaca Streptococcus dysgalactiae subspecies dysgalactiae (patógeno animal), Streptococcus canis (excepcional en humanos) y Streptococcus dysgalactiae subspecies equisimilis (SDSE), reconocido como patógeno humano y con varias publicaciones que mencionan su alta incidencia como agente de enfermedad invasora, especialmente en adultos mayores $^{1,2}$.

Presentamos el caso clínico de una niña internada en nuestro centro por una enfermedad invasora por SDSE.

\section{Caso clínico}

Paciente de sexo femenino, con 6 años de edad, y los antecedentes de síndrome de Down, hipotiroidismo en tratamiento y cardiopatía congénita operada en su primer año de vida (canal aurículo-ventricular y ductus arterioso persistente). Tenía las vacunas del Programa Nacional de Inmunizaciones (PNI) al día, sin vacunas extra-PNI.

Consultó dos veces en el Servicio de Urgencia Infantil (SUI) a los cuatro y cinco días de evolución de un cuadro de fiebre y eritrodermia. El eritema comenzó en una hemi-cara y luego se extendió al lado contra-lateral. Al segundo día presentó fiebre hasta $40^{\circ} \mathrm{C}$, con aumento del eritema en progresión descendente, que comprometió el cuello, tronco y ambas extremidades superiores. Además se acompañó de la aparición de tres flictenas eritematosas, no pruriginosas, en la región mentoniana y preauricular que desaparecieron espontáneamente en el transcurso del día. Se evaluó con exámenes de laboratorio, destacando un hemograma con recuento de leucocitos normales $\left(10.470 / \mathrm{mm}^{3}\right)$ con predominio polimorfonuclear $(92,3 \%)$, sin alteración de la serie roja y proteína $\mathrm{C}$ reactiva $(\mathrm{PCR})$ de $6,5 \mathrm{mg} / \mathrm{dl}(\mathrm{VN}<1 \mathrm{mg} / \mathrm{dl})$. Se le hizo el diagnóstico de una dermatitis alérgica y se indicó clorfenamina y glucocorticoides vía oral, durante tres días.

Por persistencia de la sintomatología, consultó a su pediatra tratante quien lo derivó nuevamente al SUI.
Hospital Clínico San Borja Arriarán.

Programa de Especialización en Pediatría, Universidad de Chile (MAP, GH).

Servicio de Pediatría (LD)

Sin conflictos de interés.

Sin fuentes de financiamiento externo.

Recibido: 17 de marzo de 2014 Aceptado: 19 de agosto de 2014

Correspondencia a: Miguel A. Pantoja mpantoja86@gmail.com 
Figura 1. Eritrodermia de tronco y abdomen superior. El eritema respeta la porción distal de ambas extremidades superiores.

Figura 2. Se observa compromiso más difuso de región cervical posterior y dorsal superior, de bordes menos delimitados.

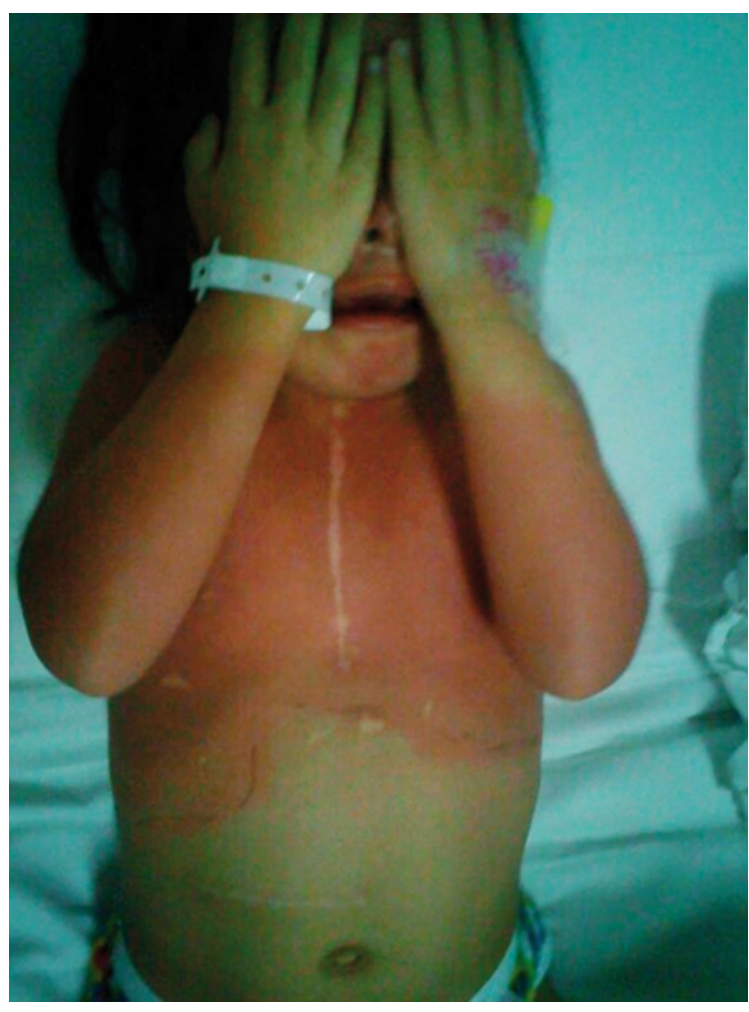

Se solicitaron exámenes de laboratorio, destacando un hemograma con leucopenia de $4.880 / \mathrm{mm}^{3}$, con predominio polimorfonuclear $(90 \%)$, plaquetas $198.000 / \mathrm{mm}^{3}$, PCR de $20,2 \mathrm{mg} / \mathrm{dl}$ y anticuerpos anti-estreptolisina $\mathrm{O} 759 \mathrm{U} /$ $\mathrm{ml}(\mathrm{VN}:<200)$. Además se tomaron un cultivo faríngeo y dos hemocultivos periféricos.

Ingresó al Servicio de Pediatría al $8^{\circ}$ día de evolución, en buenas condiciones generales, con estabilidad hemodinámica y sin compromiso de conciencia. Se planteó el diagnóstico de escarlatina e inició tratamiento antimicrobiano con amoxicilina, vía oral. Además se planteó la necesidad de realizar un ecocardiograma para descartar una enfermedad de Kawasaki atípica o una endocarditis infecciosa. Se controlaron exámenes a las $24 \mathrm{~h}$ de su ingreso. Tenía una PCR de $23,9 \mathrm{mg} / \mathrm{dl}$ y un perfil bioquímico normal. Evaluada por infectología, se decidió cambiar tratamiento antimicrobiano a penicilina sódica i.v.

Evolucionó desde el inicio del tratamiento antimicrobiano, en buenas condiciones generales. A las 48 $\mathrm{h}$ de incubación se informaron los dos hemocultivos positivos para Streptococcus $\beta$ - hemolítico del grupo G, con susceptibilidad in vitro a penicilina, clindamicina, cloxacilina, eritromicina y levofloxacino, y resistencia a cotrimoxazol. El ecocardiograma realizado fue normal y no hubo detección de otros focos clínicos secundarios.

Evolucionó afebril, con franca disminución del eritema

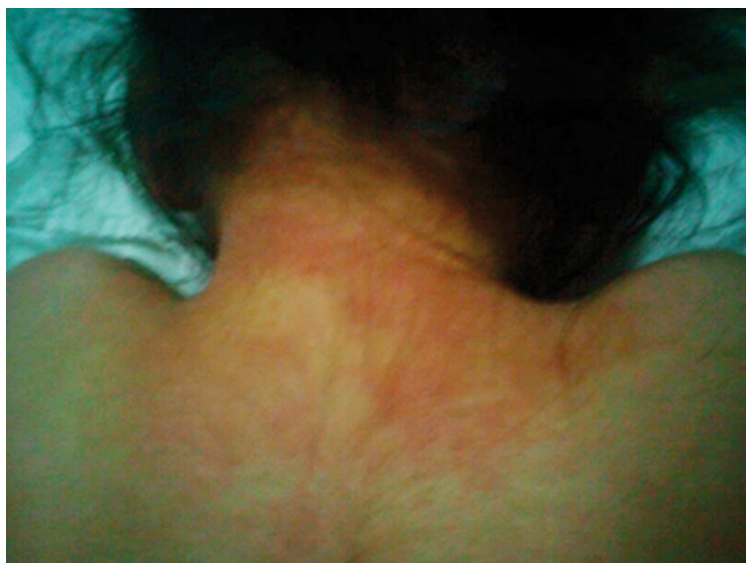

En ese momento, en su $8^{\circ}$ día de evolución y $7^{\circ}$ día de fiebre, se apreciaba en buenas condiciones generales. Al examen físico se describía una "lesión eritro-descamativa en la cara que respetaba la zona peribucal y una placa eritematosa en las extremidades superiores que respetaba las muñecas, manos y cara anterior de codo. También afectaba el cuero cabelludo, cuello y mitad superior de tronco, con bordes bien delimitados, sin adenopatías palpables. Presentaba una inyección conjuntival sin secreción y lengua aframbuesada. El examen cardíaco era normal, y no presentaba descamación periungueal" (Figuras 1 y 2). facial y de tronco. $\mathrm{Al} 5^{\circ}$ día de tratamiento antimicrobiano, se informó que la especie identificada, por método de Vitek ${ }^{\circledR}$, correspondía a Streptococcus dysgalactiae subespecie equisimilis.

La paciente recibió 10 días de tratamiento antimicrobiano i.v. más cuatro días de amoxicilina/ácido clavulánico, vía oral, completando en la fase ambulatoria 14 días de tratamiento. El control posterior demostró una evolución satisfactoria. Se realizó un estudio básico de inmunodeficiencia descartándose una hipogamaglobulinemia, hipocomplementemia o leucopenia secundaria al Síndrome de Down.

La cepa se envió al Laboratorio de Biología Molecular de la Universidad Católica para genotipificación. Por técnica de RPC universal se confirmó Streptococcus dysgalactiae subespecie equisimilis, genotipo stg653.0.

\section{Discusión}

El caso clínico descrito, una escolar con síndrome de Down que cursó con una enfermedad invasora por SDSE y una eritrodermia como forma de presentación, es el primer caso publicado de este agente en niños en nuestro país.

Dentro del grupo de Streptococcus $\beta$-hemolíticos aislados clínicamente, S. pyogenes y $S$. agalactiae fueron considerados los principales patógenos humanos hasta principios de la década de los 90. Sin embargo, estudios 
recientes de incidencia de enfermedad invasora por Streptococcus $\beta$-hemolíticos no A o B, han demostrado que la frecuencia de estos microorganismos ha alcanzado una incidencia similar a $S$. pyogenes en la población general, como lo muestra el estudio de Broyle ${ }^{1}$.

Dentro del grupo de Streptococcus $\beta$-hemolíticos no A o B se encuentran los grupos C, G, F y L de Lancefield; siendo los grupos $\mathrm{C}$ y $\mathrm{G}$ los más comunes. De acuerdo a recientes estudios taxonómicos, colonias de Streptococcus de los grupos $\mathrm{C}$ y $\mathrm{G}$ que infectan a humanos, previamente denominados Streptococcus equisimilis, fueron clasificados como SDSE ${ }^{3}$.

Streptococcus dysgalactiae subespecie equisimilis posee antígenos capsulares $\mathrm{A}, \mathrm{C}$ o $\mathrm{G}$ de la clasificación de Lancefield y presenta una marcada $\beta$-hemólisis en agar sangre $^{2}$. Comparte una gran similitud en su genoma con $S$. pyogenes (hasta $63 \%)^{4}$ en relación a los factores de virulencia. Dentro de éstos, se encuentran estreptolisina $\mathrm{O}$ y S; super-antígenos (SpeA, SpeC, SpeM) e inactivadores del complemento (C5a peptidasa y estreptoquinasa) ${ }^{5}$ (Tabla 1). Recientemente se ha descrito la presencia de una bacteriocina llamada disgalacticina, que estaría dirigida contra $S$. pyogenes, otorgándole una ventaja ecológica a SDSE en el hospedero.

Streptococcus dysgalactiae subspecies equisimilis coloniza el tracto respiratorio superior, gastrointestinal, genital femenino y ocasionalmente la piel. Su transmisión es por contacto persona-persona y las infecciones zoonóticas son comparativamente infrecuentes. Se han descrito brotes comunitarios e intrahospitalarios ${ }^{5}$.

En el ser humano, SDSE causa enfermedades superficiales o profundas, mediadas por toxinas o por mecanismos inmunológicos. Éstas van desde infecciones cutáneas superficiales a patologías potencialmente letales como el síndrome de shock tóxico estreptocóccico. La presentación clásica del adulto es secundaria a una faringitis, lo que también puede manifestarse en niños. Además puede presentarse como infecciones de piel o tejidos blandos como celulitis, erisipela, abscesos y fascitis necrosante. En cuanto a la enfermedad invasora, existen casos descritos de compromiso óseo, renal, meníngeo, abscesos abdominales, endocarditis, sepsis puerperal o neonatal y shock tóxico.

Existen actualmente varios estudios, tanto en pacientes pediátricos como adultos, sobre enfermedad invasora por SDSE. Broyle ${ }^{1}$ realizó un estudio poblacional para determinar la epidemiología de la enfermedad invasora por Streptococcus no A ni B. Describe una incidencia de $3,2 \times 100.000$ habitantes en la población general, 80\% corresponde a SDSE y $17 \%$ a Streptococcus del grupo anginosus. Sin embargo, al observar la distribución por edad, en población menor de 20 años se observó $15 \%$ de SDSE (tres casos) versus $84 \%$ de Streptococcus del grupo anginosus.
Tabla 1. Factores de virulencia de Streptococcus $\beta$-hemolíticos

\begin{tabular}{|c|c|c|c|}
\hline Proteínas con secuencias altamente similares & SDSE & S. pyogenes & S. agalactiae \\
\hline Proteínas de unión a fibronectina & $x$ & $x$ & - \\
\hline Proteínas de unión a plasminógeno & $x$ & $x$ & $x$ \\
\hline Gliceraldehido-3-fosfato deshidrogenasa & $x$ & $x$ & $x$ \\
\hline Enolasa estreptocóccica de superficie & $x$ & $x$ & $x$ \\
\hline Vitronectina & $x$ & $x$ & $x$ \\
\hline Proteínas de unión a laminina & $x$ & $x$ & $x$ \\
\hline Estreptolisina $\mathrm{O}$ & $x$ & $x$ & - \\
\hline Estreptolisina S & $x$ & $x$ & - \\
\hline Superantígenos (speA, Spec, SpeG, SpeM, Ssa, Smez) & $x$ & $x$ & - \\
\hline Disgalacticina & $x$ & - & - \\
\hline Proteína M & $x$ & $x$ & - \\
\hline Cápsula & $x$ & $x$ & $x$ \\
\hline C5a peptidasa & $x$ & $x$ & $x$ \\
\hline Proteína G & $x$ & - & - \\
\hline Estreptoquinasa & $x$ & $x$ & - \\
\hline
\end{tabular}

(Adaptado de Hughes, 2009) (Referencia 3). SDSE: Streptococcus dysgalactiae subespecie equisimilis.

Liao y cols., publicaron una serie de casos de bacteriemia por Streptococcus del grupo G. Describieron 106 episodios en 92 pacientes en un período de seis años, siendo SDSE identificado en 94\% de los casos; sin embargo, la gran mayoría correspondía a población adulta con sólo un caso de bacteriemia por Streptococcus grupo G (no especificado) en un niño ${ }^{7}$. Lopardo y cols., reportaron 95 casos de enfermedad invasora por $S$. pyogenes y SDSE en 74 pacientes, de los cuales $49 \%$ (36 casos) eran niños. De este grupo, tres casos $(8,3 \%)$ correspondían a SDSE, con un paciente fallecido (neonato) ${ }^{8}$.

Takahashi y cols., realizaron un estudio multicéntrico en Japón, que comparó las características clínicas de las infecciones por Streptococcus $\beta$-hemolíticos en un período de un año. Se aislaron 231 cepas de SDSE, ninguna de pacientes pediátricos. Al comparar la presentación con $S$. pyogenes y $S$. agalactiae, se encontró una diferencia estadísticamente significativa en la edad de presentación, siendo mayor en las infecciones por SDSE, con una mediana de 75 años 9 .

Dos estudios de colonización faríngea en población pediátrica en zonas con altas tasas de enfermedad estreptocóccica y post- estreptocóccica mostraron una tasa de colonización de SDSE hasta ocho veces mayor que por $S$. pyogenes. Sin embargo, los casos de faringitis estreptocóccica fueron en su mayoría provocadas por $S$. pyogenes, lo que abre un debate en relación al rol patogénico de SDSE en la enfermedad estreptocóccica y post-estreptocóccica ${ }^{4,10}$. 
El diagnóstico microbiológico se realiza por la evidencia de hemólisis en placas de agar sangre y la identificación de los antígenos C y G. Sin embargo, hay que diferenciarlo de Streptococcus del grupo anginosus, lo que se logra con la reacción de Voges-Proskauer. También se ha descrito SDSE con antígeno A de Lancefield, donde la ausencia de producción de pirrolidonilarilamidasa es característica. Los estudios automatizados como el sistema Vitek ${ }^{\circledR}$ pueden identificar de manera confiable $94 \%$ de las muestras. Sin embargo, la identificación de manera más segura, como se realizó con la cepa aislada del caso descrito, se logra a través de métodos de biología molecular con el análisis del ARNr 16s que permite tipificar la bacteria usando los genes emm. Para esto se emplea una base de datos del CDC de E.U.A.

En un estudio en Japón, se encontraron diferencias significativas en relación a la genotipificación emm, al comparar los agentes aislados de pacientes con enfermedad invasora (stG485, stG6792 y stG2078) versus no invasora ${ }^{11}$.

Respecto del tratamiento, los $\beta$-lactámicos, y específicamente penicilina, es el antimicrobiano de elección. Debido a su mantenida susceptibilidad. El uso de amikacina ha sido propuesto en infecciones graves por su acción sinérgica con los $\beta$-lactámicos, y en casos de shock tóxico se recomienda asociar clindamicina e inmunoglobulina intravenosa. Se describe resistencia a macrólidos (16$24 \%$ ), a tetraciclinas $(60 \%)$ y existen algunos reportes de resistencia a fluoroquinolonas. No se ha descrito resistencia a vancomicina o linezolid ${ }^{5}$.

En conclusión, SDSE es un agente frecuente de enfermedades del adulto; en pacientes pediátricos sólo es responsable de enfermedad invasora en casos excepcionales. El diagnóstico por métodos automatizados resulta adecuado y el tratamiento de primera línea es penicilina G. Son necesarios nuevos estudios para determinar la relevancia de la portación faríngea de SDSE en países de alta prevalencia de enfermedad post-estreptocóccica.

\section{Resumen}

Las infecciones por estreptococos piógenos, comunes en la edad pediátrica, muestran en los últimos años un aumento en la identificación de grupos no A o B. Presentamos el caso de una niña de 6 años, con historia de fiebre de cinco días de evolución asociado a una lesión eritrodérmica con hemocultivos positivos a Streptococcus dysgalactiae subspecies equisimilis (confirmado por biología molecular-genotipificación). Recibió terapia antimicrobiana por 14 días con $\beta$-lactámicos con una evolución favorable. Este estreptococo $\beta$-hemolítico, presenta antígenos del grupo A, C y G de Lancefield y una gran similitud con Streptococcus pyogenes en relación a los factores de virulencia. Más frecuentemente aislado en adultos mayores, existen pocos casos descritos en población pediátrica. El tratamiento de primera línea es con $\beta$-lactámicos, para los cuales no hay reportes de resistencia antimicrobiana.

\section{Referencias bibliográficas}

1.- Broyles L N, Van Beneden C, Beall B, Facklam R, Shewmaker P L, Malpiedi P, et al. Population-based study of invasive disease due to beta-hemolytic streptococci of groups other than A and B. Clin Infect Dis 2009; 48 706-12.

2.- Takahashi T, Ubukata K, Watanabe H. Invasive infection caused by Streptococcus dysgalactiae subsp. equisimilis: characteristics of strains and clinical features. J Infect Chemother 2011; 17: 1-10.

3.- Facklam R. What happened to the streptococci: overview of taxonomic and nomenclature changes. Clin Microbiol Rev 2002; 15: 613-30.

4.- McDonald M, Towers R J, Andrews R M, Carapetis J R, Currie B J. Epidemiology of Streptococcus dysgalactiae subsp. equisimilis in tropical communities, Northern Australia. Emerg Infect Dis 2007; 13: 1694-700.
5.- Brandt C, Spellerberg B. Human infections due to Streptococcus dysgalactiae subspecies equisimilis. Clin Infect Dis 2009; 49: 766-72.

6.- Heng N C, Ragland N L, Swe P M, Baird H J, Inglis M A, Tagg J R, et al. Dysgalacticin: a novel, plasmid-encoded antimicrobial protein (bacteriocin) produced by Streptococcus dysgalactiae subsp. equisimilis. Microbiology 2006; 152: 1991-2001.

7.- Liao C H, Liu L C, Huang Y T, Teng L J, Hsueh P R. Bacteremia caused by Group G streptococci, Taiwan. Emerg Infect Dis 2008; 14: 837-40.

8.- Lopardo H A, Vidal P, Sparo M, Jeric P, Centron D, Facklam R R, et al. Six-month multicenter study on invasive infections due to Streptococcus pyogenes and Streptococcus dysgalactiae subsp. equisimilis in Argentina. J Clin Microbiol 2005; 43: 802-7.

9.- Takahashi T, Sunaoshi K, Sunakawa K,
Fujishima S, Watanabe H, Ubukata K. Clinical aspects of invasive infections with Streptococcus dysgalactiae ssp. equisimilis in Japan: differences with respect to Streptococcus pyogenes and Streptococcus agalactiae infections. Clin Microbiol Infect 2010; 16: 1097-103.

10.- Bramhachari P V, Kaul S Y, McMillan D J, Shaila M S, Karmarkar M G, Sriprakash K S. Disease burden due to Streptococcus dysgalactiae subsp. equisimilis (group $\mathrm{G}$ and C Streptococcus) is higher than that due to Streptococcus pyogenes among Mumbai school children. J Med Microbiol 2010; 59: 220-3.

11.- Sunaoshi K, Murayama S Y, Adachi K, Yagoshi M, Okuzumi K, Chiba N, et al. Molecular emm genotyping and antibiotic susceptibility of Streptococcus dysgalactiae subsp. equisimilis isolated from invasive and non-invasive infections. J Med Microbiol 2010; 59: $82-8$. 